\title{
Spectral parameter power series for Sturm-Liouville problems
}

\author{
Vladislav V. Kravchenko and R. Michael Porter \\ Department of Mathematics, CINVESTAV del IPN, Unidad Querétaro \\ Libramiento Norponiente No. 2000, Fracc. Real de Juriquilla \\ Queretaro, Qro. C.P. 76230 MEXICO \\ e-mail: vkravchenko@qro.cinvestav.mx; mike@math.cinvestav.mx
}

October 24, 2018

\begin{abstract}
We consider a recently discovered representation for the general solution of the Sturm-Liouville equation as a spectral parameter power series (SPPS). The coefficients of the power series are given in terms of a particular solution of the Sturm-Liouville equation with the zero spectral parameter. We show that, among other possible applications, this provides a new and efficient numerical method for solving initial value and boundary value problems. Moreover, due to its convenient form the representation lends itself to numerical solution of spectral Sturm-Liouville problems, effectively by calculation of the roots of a polynomial. We discuss examples of the numerical implementation of the SPPS method and show it to be equally applicable to a wide class of singular Sturm-Liouville problems as well as to problems with spectral parameter dependent boundary conditions.
\end{abstract}

\section{Introduction}

In the recent work [14 a representation was obtained for solutions of the equation

$$
\left(p u^{\prime}\right)^{\prime}+q u=\lambda u
$$

in terms of a known non-trivial solution of the equation

$$
\left(p u_{0}^{\prime}\right)^{\prime}+q u_{0}=0,
$$

where $p, q, u, u_{0}$ are complex-valued functions of the real variable $x$ satisfying certain smoothness conditions, and $\lambda$ is an arbitrary complex constant. The general solution of (1) has the form of a power series with respect to the spectral parameter $\lambda$. The fact that under certain conditions $u$ is a complex analytic 
function of $\lambda$ has long been known (see, e.g., [16]). The representation from [14] gives a simple procedure for constructing its Taylor coefficients.

Our main purpose here is to show that this representation gives us, among other possible applications, a simple and powerful method for numerical solution of initial value, boundary value and spectral problems. We will generalize slightly by considering the equation

$$
\left(p u^{\prime}\right)^{\prime}+q u=\lambda r u
$$

and representing its general solution in terms of a solution of (2), which does not depend on the coefficient $r$.

In Section 1 we prove the main theorem and make several observations which will apply to the subsequent applications. In Section 2 we observe that the rate of convergence of the spectral parameter power series (SPPS) corresponding to (3) is quite easy to estimate. This fact and the simple form of the solutions make the SPPS method appropriate for solving initial and boundary value problems. Perhaps more importantly, the representation of a solution of the Sturm-Liouville equation in the form of an SPPS is well-suited for solving spectral problems as it reduces problem to calculation of zeros of an analytic function defined by its Taylor series. In Section 3 we show that even difficult examples like the Coffey-Evans potential can be handled by the SPPS method; there is little doubt that the numerical performance of the method can be significantly improved using additional computational techniques discussed in the paper. In Section 4 we show that the appearance of the spectral parameter in the boundary conditions does not provoke additional difficulties in application of the SPPS method. As an example of application of the SPPS method to singular Sturm-Liouville problems we consider in Section [5 a very interesting example of a highly non-self-adjoint operator (with complex and singular coefficients) discussed in a number of recent publications [2, 3, 7, 10, 11, 21, 22. The SPPS method makes it possible to obtain eigenvalues and eigenfunctions of the problem even when other known algorithms encounter numerical difficulties.

\section{Solution of the Sturm-Liouville equation}

Here we formulate a generalization of the main result of [14, which allows us to obtain a general solution of the Sturm-Liouville equation (3) in the form of a spectral parameter power series. The proof does not depend on pseudoanalytic function theory (which was the tool employed in [14]).

Theorem 1 Assume that on a finite interval $[a, b]$, equation (2) possesses a particular solution $u_{0}$ such that the functions $u_{0}^{2} r$ and $1 /\left(u_{0}^{2} p\right)$ are continuous on $[a, b]$. Then the general solution of (3) on $(a, b)$ has the form

$$
u=c_{1} u_{1}+c_{2} u_{2}
$$


where $c_{1}$ and $c_{2}$ are arbitrary complex constants,

$$
u_{1}=u_{0} \sum_{k=0}^{\infty} \lambda^{k} \tilde{X}^{(2 k)} \quad \text { and } \quad u_{2}=u_{0} \sum_{k=0}^{\infty} \lambda^{k} X^{(2 k+1)}
$$

with $\widetilde{X}^{(n)}$ and $X^{(n)}$ being defined by the recursive relations

$$
\begin{gathered}
\widetilde{X}^{(0)} \equiv 1, \quad X^{(0)} \equiv 1, \\
\widetilde{X}^{(n)}(x)= \begin{cases}\int_{x_{0}}^{x} \widetilde{X}^{(n-1)}(s) u_{0}^{2}(s) r(s) d s, & n \text { odd }, \\
\int_{x_{0}}^{x} \widetilde{X}^{(n-1)}(s) \frac{1}{u_{0}^{2}(s) p(s)} d s, & n \text { even },\end{cases} \\
X^{(n)}(x)= \begin{cases}\int_{x_{0}}^{x} X^{(n-1)}(s) \frac{1}{u_{0}^{2}(s) p(s)} d s, & n \text { odd }, \\
\int_{x_{0}}^{x} X^{(n-1)}(s) u_{0}^{2}(s) r(s) d s, & n \text { even },\end{cases}
\end{gathered}
$$

where $x_{0}$ is an arbitrary point in $[a, b]$ such that $p$ is continuous at $x_{0}$ and $p\left(x_{0}\right) \neq 0$. Further, both series in (5) converge uniformly on $[a, b]$.

Proof. First we prove that $u_{1}$ and $u_{2}$ are indeed solutions of (3) whenever the application of the operator $L=\frac{d}{d x} p \frac{d}{d x}+q$ to them makes sense. For this, note that if $L u_{0}=0$, then $L$ can be written in the factorized form $L=\frac{1}{u_{0}} \frac{d}{d x} p u_{0}^{2} \frac{d}{d x} \frac{1}{u_{0}}$. Then application of $\frac{1}{r} L$ to $u_{1}$ gives

$$
\begin{aligned}
\frac{1}{r} L u_{1} & =\frac{1}{r u_{0}} \frac{d}{d x}\left(p u_{0}^{2} \frac{d}{d x} \sum_{k=0}^{\infty} \lambda^{k} \widetilde{X}^{(2 k)}\right)=\frac{1}{r u_{0}} \frac{d}{d x} \sum_{k=1}^{\infty} \lambda^{k} \widetilde{X}^{(2 k-1)} \\
& =u_{0} \sum_{k=1}^{\infty} \lambda^{k} \widetilde{X}^{(2 k-2)}=\lambda u_{1} .
\end{aligned}
$$

In a similar way one can check that $u_{2}$ satisfies (3) as well. In order to give sense to this chain of equalities it is sufficient to prove the uniform convergence of the series involved in $u_{1}$ and $u_{2}$ as well as of the series obtained by a term-wise differentiation. This can be done with the aid of the Weierstrass M-test. Indeed, we have $\left|\widetilde{X}^{(2 k)}\right| \leq\left(\max \left|r u_{0}^{2}\right|\right)^{k}\left(\max \left|\frac{1}{p u_{0}^{2}}\right|\right)^{k} \frac{|b-a|^{2 k}}{(2 k) !}$ and the series $\sum_{k=0}^{\infty} \frac{c^{k}}{(2 k) !}$ is convergent where

$$
c=|\lambda|\left(\max \left|r u_{0}^{2}\right|\right)\left(\max \left|\frac{1}{p u_{0}^{2}}\right|\right)|b-a|^{2} .
$$


The uniform convergence of the series in $u_{2}$ as well as of the series of derivatives can be shown similarly.

The last step is to verify that the Wronskian of $u_{1}$ and $u_{2}$ is different from zero at least at one point (which necessarily implies the linear independence of $u_{1}$ and $u_{2}$ on the whole segment $[a, b]$ ). It is easy to see that by definition all the $\widetilde{X}^{(n)}\left(x_{0}\right)$ and $X^{(n)}\left(x_{0}\right)$ vanish except for $\widetilde{X}^{(0)}\left(x_{0}\right)$ and $X^{(0)}\left(x_{0}\right)$ which equal 1. Thus

$$
\begin{aligned}
& u_{1}\left(x_{0}\right)=u_{0}\left(x_{0}\right), \quad u_{1}^{\prime}\left(x_{0}\right)=u_{0}^{\prime}\left(x_{0}\right), \\
& u_{2}\left(x_{0}\right)=0, \quad u_{2}^{\prime}\left(x_{0}\right)=\frac{1}{u_{0}\left(x_{0}\right) p\left(x_{0}\right)}
\end{aligned}
$$

and the Wronskian of $u_{1}$ and $u_{2}$ at $x_{0}$ equals $1 / p\left(x_{0}\right) \neq 0$.

Remark 2 In the case $\lambda=0$, the solution (5) becomes $u_{1}=u_{0}$ and $u_{2}=$ $u_{0} \int_{x_{0}}^{x} \frac{d s}{u_{0}^{2}(s) p(s)}$. The expression for $u_{2}$ is a well known formula for constructing a second linearly independent solution.

Remark 3 The result of Theorem 1 is valid for infinite intervals as well, the series being uniformly convergent on any finite subinterval.

Remark 4 One of the functions $r u_{0}^{2}$ or $1 /\left(p u_{0}^{2}\right)$ may not be continuous on $[a, b]$ and yet $u_{1}$ or $u_{2}$ may make sense. For example, in the case of the Bessel equation $\left(x u^{\prime}\right)^{\prime}-\frac{1}{x} u=-\lambda x u$, we can choose $u_{0}(x)=x / 2$. Then $1 /\left(p u_{0}^{2}\right) \notin C[0,1]$. Nevertheless all integrals in (7) exist and $u_{1}$ coincides with the nonsingular $J_{1}(\sqrt{\lambda} x)$, while $u_{2}$ is a singular solution of the Bessel equation.

Remark 5 In the regular case the existence and construction of the required $u_{0}$ presents no difficulty. Let $p$ and $q$ be real valued, $p(x) \neq 0$ for all $x \in[a, b]$ and let $p, p^{\prime}, r$ and $q$ be continuous on $[a, b]$. Then (2) possesses two linearly independent regular solutions $v_{1}$ and $v_{2}$ whose zeros alternate. Thus one may choose $u_{0}=v_{1}+i v_{2}$.

Even when the coefficient functions are not real-valued, nonvanishing solutions abound. Assume that the equation possesses a pair of linearly independent solutions $v_{1}$ and $v_{2}$. Let $A_{j}=\left\{x \in(a, b): v_{j}(x) \neq 0\right\}, j=1,2$. The function $\psi(c, x)=c v_{1}(x)+v_{2}(x)$ for $c \in \mathbb{C}, x \in A_{1}$, has maximal (real) rank 2 (i.e., $\psi$ is a submersion) if and only if $v_{1}(x) \neq 0$. By the preimage theorem [13, chapter 1], the preimage $\psi^{-1}(0)$ is a 1-dimensional submanifold of $\mathbb{C} \times A_{1}$ and therefore its projection $B_{1} \subseteq \mathbb{C}$ has zero measure. For every $c \in \mathbb{C} \backslash B_{1}$, the linear combination $c v_{1}+v_{2}$ does not vanish in $A_{1}$. The same reasoning gives us a null set $B_{2}$ such that $v_{1}+c v_{2}$ does not vanish in $A_{2}$ when $c \in \mathbb{C} \backslash B_{2}$. Altogether, there exist nonvanishing solutions throughout $A_{1} \cup A_{2}$, which is all of $(a, b)$ because $v_{1}, v_{2}$ are linearly independent solutions of a second order differential equation. 
Remark 6 The procedure for construction of solutions described in Theorem 1 works not only when a solution is available for $\lambda=0$, but in fact when a solution of the equation

$$
\left(p u_{0}^{\prime}\right)^{\prime}+q u_{0}=\lambda_{0} r u_{0}
$$

is known for some fixed $\lambda_{0}$. The solution (5) now takes the form

$$
u_{1}=u_{0} \sum_{k=0}^{\infty}\left(\lambda-\lambda_{0}\right)^{k} \tilde{X}^{(2 k)} \quad \text { and } \quad u_{2}=u_{0} \sum_{k=0}^{\infty}\left(\lambda-\lambda_{0}\right)^{k} X^{(2 k+1)} .
$$

This can be easily verified by writing (3) as

$$
\left(L-\lambda_{0} r\right) u=\left(\lambda-\lambda_{0}\right) r u .
$$

The operator on the left-hand side can be factorized exactly as in the proof of the theorem, and the same reasoning carries through.

Remark 7 For calculating the series in (5) it may be convenient to calculate $X^{(n)}$ or $\widetilde{X}^{(n)}$ directly from $X^{(n-2)}$ or $\widetilde{X}^{(n-2)}$. For example, when $n$ is even we have

$$
\begin{aligned}
\widetilde{X}^{(n)}(x) & =\int_{x_{0}}^{x} \frac{1}{u_{0}(s)^{2} p(s)} \int_{x_{0}}^{s} u_{0}(t)^{2} r(t) \widetilde{X}^{(n-2)}(t) d t d s \\
& =\int_{x_{0}}^{x}(P(x)-P(t)) u_{0}(t)^{2} r(t) \widetilde{X}^{(n-2)}(t) d t
\end{aligned}
$$

where $P^{\prime}=1 /\left(u_{0}^{2} p\right)$.

Remark 8 Other representations of the general solution of (3) as a formal power series have been long known (see [17. Theorem 1], [4]) and used for studying qualitative properties of solutions. The complicated manner in which the parameter $\lambda$ appears in those representations makes that form of a general solution too difficult for quantitative analysis of spectral and boundary value problems. In contrast, the solution (4)-(8) is a power series with respect to $\lambda$, making it quite attractive for numerical solution of spectral, initial value and boundary value problems.

A special case of Theorem 1 with $q \equiv 0, \lambda=1$, was known to $\mathrm{H}$. Weyl.

Corollary 9 ([23]) Let $1 / p$ and $r$ be continuous on $[a, b]$. The general solution of the equation

$$
\left(p u^{\prime}\right)^{\prime}=r u
$$

on $(a, b)$ has the form

$$
u=c_{1} u_{1}+c_{2} u_{2}
$$

where $c_{1}$ and $c_{2}$ are arbitrary constants and $u_{1}, u_{2}$ are defined by (5)-(8) with $u_{0} \equiv \lambda=1$.

This corollary enables us to find the particular solution $u_{0}$ discussed in Re$\operatorname{mark}$ 5. 


\section{Numerical solution of initial value problems}

Consider the Sturm-Liouville equation (3) on $[a, b]$ with any desired initial conditions. The numerical implementation of the solution via the representation (5) for a general solution is algorithmically simple. One must consider the accuracy of calculation of the iterated integrals in (7) and (8), and the rate of convergence of the series (5), because in numerical work one must work with finitely many terms.

The main parameters that one can control are the number $M$ of subintervals in which to divide $[a, b]$ when integrating numerically and the number $N$ of powers in the truncated series. The relationship of $M$ to the accuracy of the integrals is a standard question and will not be discussed here. In regards to $N$, observe that one can not always expect a good approximation to $u$ over all of $[a, b]$ with a series of $N$ terms, no matter how precisely the integrals are calculated. However, using the estimate for $\left|\widetilde{X}^{(2 k)}\right|$ and $\left|X^{(2 k-1)}\right|$ (see the proof of Theorem 1 below) it is easy to obtain a rough but useful estimate for the tail of the SPPS. Namely, consider $\left|u_{1}-u_{1, N}\right|$ where $u_{1, N}=u_{0} \sum_{k=0}^{N} \lambda^{k} \widetilde{X}^{(2 k)}$. We have

$$
\begin{aligned}
\left|u_{1}-u_{1, N}\right| & =\left|u_{0}\right|\left|\sum_{k=N+1}^{\infty} \lambda^{k} \tilde{X}^{(2 k)}\right| \leq \max \left|u_{0}\right| \sum_{k=N+1}^{\infty} \frac{c^{k}}{(2 k) !} \\
& =\max \left|u_{0}\right|\left|\cosh \sqrt{c}-\sum_{k=0}^{N} \frac{c^{k}}{(2 k) !}\right|
\end{aligned}
$$

where $c$ is defined by (9). In a similar way one can see that the remainder of the SPPS corresponding to $u_{2}$ is estimated by the tail of the power series of $\sinh \sqrt{c}$. Thus, if a certain value of $N$ is seen to be insufficient for achieving a required accuracy, the interval can be subdivided and the initial value problem solved on the first subinterval. The initial values of the solution for the second subinterval are calculated easily taking into account that $u_{1}^{\prime}=\frac{u_{0}^{\prime}}{u_{0}} u_{1}+\frac{1}{u_{0} p} \sum_{k=1}^{\infty} \lambda^{k} \widetilde{X}^{(2 k-1)}$ (and analogously for $u_{2}$ ). Thus, no numerical differentiation is necessary and this process can be continued with little loss in accuracy.

The required particular solution $u_{0}$ may be calculated using any available algorithm; in the examples presented below we have applied the formula of Corollary 9, applying the above subdivision procedure. All of the calculations were performed with Mathematica (Wolfram).

\section{Spectral problems}

The fact that spectral Sturm-Liouville problems are related to the problem of finding zeros of complex analytic functions of the variable $\lambda$ is quite well 
known (see, e.g., [16]). For a regular Sturm-Liouville problem the corresponding analytic function is even entire. The representation (4)-(8) allows us to obtain the Taylor series of that analytic function explicitly. As an example, let us first consider a spectral problem for (3) with the boundary conditions

$$
u(0)=0 \quad \text { and } \quad u(1)=0 .
$$

We suppose that the coefficients satisfy the conditions from Remark 5 and that $u_{0}$ is constructed as described there, taking $x_{0}=0$. From the first boundary condition and (10), the constant $c_{1}$ in (4) must be zero. Then the spectral problem reduces to finding values of $\lambda$ for which $u_{2}(1)=u_{0}(1) \sum_{k=0}^{\infty} \lambda^{k} X^{(2 k+1)}(1)$ vanishes. In other words, this spectral problem reduces to the calculation of zeros of the complex analytic function $\kappa(\lambda)=\sum_{m=0}^{\infty} a_{m} \lambda^{m}$ where

$$
a_{m}=u_{0}(1) X^{(2 k+1)}(1) .
$$

Now let $\alpha$ and $\beta$ be arbitrary real numbers and consider the more general boundary conditions

$$
\begin{aligned}
& u(a) \cos \alpha+u^{\prime}(a) \sin \alpha=0 \\
& u(b) \cos \beta+u^{\prime}(b) \sin \beta=0
\end{aligned}
$$

together with equation (3). Taking the solutions $u_{1}$ and $u_{2}$ defined by (5) and using (10), (11) with $x_{0}=a$, we obtain from (16) the following equation,

$$
c_{1}\left(u_{0}(a) \cos \alpha+u_{0}^{\prime}(a) \sin \alpha\right)+c_{2} \frac{\sin \alpha}{u_{0}(a) p(a)}=0
$$

which gives $c_{2}=\gamma c_{1}$ when $\alpha \neq \pi n$, with $\gamma=-u_{0}(a) p(a)\left(u_{0}(a) \cot \alpha+u_{0}^{\prime}(a)\right)$, whereas $c_{1}=0$ when $\alpha=\pi n$. In the latter case the result is similar to the example considered above, thus let us suppose $\alpha \neq \pi n$. From the definition of $u_{1}$ and $u_{2}$ we have

$$
u_{1}^{\prime}=\frac{u_{0}^{\prime}}{u_{0}} u_{1}+\frac{1}{u_{0} p} \sum_{k=1}^{\infty} \lambda^{k} \widetilde{X}^{(2 k-1)} \quad \text { and } \quad u_{2}^{\prime}=\frac{u_{0}^{\prime}}{u_{0}} u_{2}+\frac{1}{u_{0} p} \sum_{k=0}^{\infty} \lambda^{k} X^{(2 k)} .
$$

Then the boundary condition (17) implies that

$$
\begin{gathered}
\left(u_{0}(b) \cos \beta+u_{0}^{\prime}(b) \sin \beta\right)\left(\sum_{k=0}^{\infty} \lambda^{k} \widetilde{X}^{(2 k)}(b)+\gamma \sum_{k=0}^{\infty} \lambda^{k} X^{(2 k+1)}(b)\right) \\
+\frac{\sin \beta}{u_{0}(b) p(b)}\left(\sum_{k=1}^{\infty} \lambda^{k} \widetilde{X}^{(2 k-1)}(b)+\gamma \sum_{k=0}^{\infty} \lambda^{k} X^{(2 k)}(b)\right)=0 .
\end{gathered}
$$


Thus the spectral problem (3), (16), (17) reduces to the problem of calculating zeros of the analytic function $\kappa(\lambda)=\sum_{m=0}^{\infty} a_{m} \lambda^{m}$ where

$$
a_{0}=\left(u_{0}(b) \cos \beta+u_{0}^{\prime}(b) \sin \beta\right)\left(1+\gamma X^{(1)}(b)\right)+\frac{\gamma \sin \beta}{u_{0}(b) p(b)}
$$

and

$$
\begin{gathered}
a_{m}=\left(u_{0}(b) \cos \beta+u_{0}^{\prime}(b) \sin \beta\right)\left(\widetilde{X}^{(2 m)}(b)+\gamma X^{(2 m+1)}(b)\right) \\
+\frac{\sin \beta}{u_{0}(b) p(b)}\left(\widetilde{X}^{(2 m-1)}(b)+\gamma X^{(2 m)}(b)\right), \quad m=1,2, \ldots
\end{gathered}
$$

This reduction of a Sturm-Liouville spectral problem lends itself to a simple numerical implementation. To calculate the first $n$ eigenvalues we consider the Taylor polynomial $\kappa_{N}(\lambda)=\sum_{m=0}^{N} a_{m} \lambda^{m}$ with $N \geq n$. Thus the numerical approximation of eigenvalues of the Sturm-Liouville problem reduces to the calculation of zeros of the polynomial $\kappa_{N}(\lambda)$.

There is no need to work with zeros of only one polynomial. It is well known that in general the higher roots of a polynomial become less stable with respect to small inaccuracies in coefficients. Our spectral parameter power series method is well suited to overcome this problem and thus to calculate higher eigenvalues with a good accuracy. This is done using Remark 6. Suppose we have already calculated the eigenvalue $\lambda_{0}$ using the procedure described above as a first root of the obtained polynomial. Then for the next step we define $U_{0}=u_{1}+i u_{2}$ where $u_{1}$ and $u_{2}$ are defined by (5) with $\lambda=\lambda_{0}$. The function $U_{0}$ is then a solution of (12). We use it to obtain the eigenvalue $\lambda_{1}$ of the original problem observing that $\lambda_{1}=\Lambda_{1}+\lambda_{0}$ where $\Lambda_{1}$ is the first eigenvalue of the equation $\left(L-\lambda_{0} r\right) u=\Lambda u$ with the same boundary conditions as in the original problem. This procedure can be continued for calculating higher eigenvalues. Note that if $\lambda_{0}=0$ we should begin this shifting procedure starting with $\lambda_{1}$.

Here we discuss some numerical examples.

Paine Problem. A number of spectral problems which have become standard test cases appear in [18, 19]. As a first example we consider

$$
\begin{gathered}
p(x)=-1, \quad q(x)=\frac{1}{(x+0.1)^{2}} . \\
u(0)=0, \quad u(\pi)=0 .
\end{gathered}
$$

The eigenvalues in the following table were calculated via SPPS using integration on 10,000 subintervals for calculating $N=100$ powers of $\lambda$. These eigenvalues were found as roots of a single polynomial (i.e., the shifting of $\lambda$ as described in Remark [6 was not applied). Due to the sensitivity of the larger roots of the polynomial to errors in the coefficients, 100-digit arithmetic was used. 


\begin{tabular}{|r|c|c|}
\hline$n$ & $\lambda_{n}[19]$ & $\lambda_{n}$ SPPS \\
\hline 0 & 1.5198658211 & 1.519865821099 \\
\hline 1 & 4.9433098221 & 4.943309822144 \\
\hline 2 & 10.284662645 & 10.28466264509 \\
\hline 3 & 17.559957746 & 17.55995774633 \\
\hline 4 & 26.782863158 & 26.78286315899 \\
\hline 5 & 37.964425862 & 37.96442587941 \\
\hline 6 & 51.113357757 & 51.11335707578 \\
\hline 7 & 66.236447704 & 66.23646092491 \\
\hline 8 & 83.338962374 & 83.33879073183 \\
\hline 9 & 102.42498840 & 102.4259718823 \\
\hline 10 & 123.49770680 & 123.512483827 \\
\hline
\end{tabular}

On the basis of the above values, a new calculation was made by shifting with $\lambda^{*}=66$, resulting in the following improved approximations for the last few eigenvalues.

\begin{tabular}{|r|c|r|}
\hline$n$ & $\lambda_{n}[19]$ & $\lambda_{n}$ SPPS \\
\hline 7 & 66.236447704 & 66.23644770359 \\
\hline 8 & 83.338962374 & 83.33896237419 \\
\hline 9 & 102.42498840 & 102.42498839828 \\
\hline 10 & 123.49770680 & 123.49770680101 \\
\hline 11 & 146.55960608 & 146.55960605783 \\
\hline 12 & 171.61264485 & 171.61265439928 \\
\hline
\end{tabular}

With $\lambda^{*}=146$ and increasing the number of powers to $N=150$, the following further values were obtained.

\begin{tabular}{|r|c|c|}
\hline$n$ & $\lambda_{n}[19]$ & $\lambda_{n}$ SPPS \\
\hline 11 & 146.55960608 & 146.55586199495330 \\
\hline 12 & 171.61264485 & 171.60875781110985 \\
\hline 13 & 198.65837500 & 198.65416389844202 \\
\hline
\end{tabular}

When the number of digits for internal calculations was increased to 150 , SPPS produced the same results.

Coffey-Evans equation. This test case, defined by

$$
\begin{gathered}
p(x)=-1, \quad q(x)=-2 \beta \cos 2 x+\beta^{2} \sin ^{2} 2 x . \\
u(-\pi / 2)=0, \quad u(\pi / 2)=0,
\end{gathered}
$$

presents the challenge of distinguishing eigenvalues within the triple clusters which form as the parameter $\beta$ increases. We present results for $\beta=20,30,50$. In all cases given here the eigenvalues were obtained without shifting $\lambda$. 
$\beta=20$.

$M=10,000$ subintervals, $N=180$ powers, 100 digits of precision.

\begin{tabular}{|l|r|r|}
\hline$n$ & $\lambda_{n}[\underline{6},[15]$ & \multicolumn{1}{c|}{$\lambda_{n}$ SPPS } \\
\hline 0 & -0.00000000000000 & 0.0000000000000003 \\
\hline 1 & 77.91619567714397 & 77.9161956771439703 \\
\hline 2 & 151.46277834645663 & 151.4627783464566396 \\
\hline 3 & 151.46322365765863 & 151.4632236576586490 \\
\hline 4 & 151.46366898835165 & 151.4636689883516575 \\
\hline 5 & 220.15422983525995 & 220.1542298352599497 \\
\hline 6 & 283.0948 & 283.0948146954014377 \\
\hline 7 & 283.2507 & 283.2507437431126800 \\
\hline 8 & 283.4087 & 283.4087354034293064 \\
\hline
\end{tabular}

$\beta=30$

$M=10,000$ subintervals, $N=150$ powers, 100 digits of precision.

\begin{tabular}{|c|c|c|}
\hline$n$ & $\lambda_{n}[\mathbf{1 5}, \mathbf{1 9}]$ & $\lambda_{n}$ SPPS \\
\hline 0 & 0.00000000000000 & 0.000000000000000002 \\
\hline 1 & 117.946307662070 & 117.94630766206876 \\
\hline 2 & & 231.664928928423790 \\
\hline 3 & 231.66492931296 & 231.664928928423791 \\
\hline 4 & & 231.664930082035462 \\
\hline 5 & & 340.888299091685489 \\
\hline 6 & & 403.219684016171863 \\
\hline 7 & & 403.219684016171917 \\
\hline
\end{tabular}

$M=10,000$ subintervals, $N=150$ powers, 100 digits of precision.

\begin{tabular}{|l|c|l|}
\hline$n$ & $\lambda_{n}[19]$ & \multicolumn{1}{|c|}{$\lambda_{n}$ SPPS } \\
\hline 0 & 0.00000000000000 & 0.000000000000000003 \\
\hline 1 & 197.968726516507 & 197.96872651650729 \\
\hline 2 & & 391.807 \\
\hline 3 & 391.80819148905 & 391.810 \\
\hline 4 & & 547.1397060 \\
\hline
\end{tabular}

\section{Sturm-Liouville problems with spectral param- eter dependent boundary conditions}

In this section we consider Sturm-Liouville problems of the form

$$
\begin{gathered}
\left(p u^{\prime}\right)^{\prime}+q u=\lambda r u, \quad x \in[a, b], \\
u(a) \cos \alpha+u^{\prime}(a) \sin \alpha=0, \quad \alpha \in[0, \pi), \\
\beta_{1} u(b)-\beta_{2} u^{\prime}(b)=\varphi(\lambda)\left(\beta_{1}^{\prime} u(b)-\beta_{2}^{\prime} u^{\prime}(b)\right),
\end{gathered}
$$

where $\varphi$ is a complex-valued function of the variable $\lambda$ and $\beta_{1}, \beta_{2}, \beta_{1}^{\prime}, \beta_{2}^{\prime}$ are complex numbers. This kind of problem arises in many physical applications 
(we refer to [1] and references therein) and has been studied in a considerable number of publications 1, 5, 8, 9, 12, 20. For some special forms of the function $\varphi$ such as $\varphi(\lambda)=\lambda$ or $\varphi(\lambda)=\lambda^{2}+c_{1} \lambda+c_{2}$, results were obtained [8], [20] concerning the regularity of the problem (18)-(20); we will not dwell upon the details. Our purpose is to show the applicability of the spectral parameter power series (SPPS) method to this type of Sturm-Liouville problems. For simplicity, let us suppose that $\alpha=0$ and hence the condition (19) becomes $u(a)=0$. Then as was shown in the preceding section, if an eigenfunction exists it necessarily coincides with $u_{2}$ up to a multiplicative constant.

In this case condition (20) becomes equivalent to the equality

$$
\left(u_{0}(b) \varphi_{1}(\lambda)-u_{0}^{\prime}(b) \varphi_{2}(\lambda)\right) \sum_{k=0}^{\infty} \lambda^{k} X^{(2 k+1)}(b)-\frac{\varphi_{2}(\lambda)}{u_{0}(b) p(b)} \sum_{k=0}^{\infty} \lambda^{k} X^{(2 k)}(b)=0
$$

where $\varphi_{1,2}(\lambda)=\beta_{1,2}-\beta_{1,2}^{\prime} \varphi(\lambda)$. Calculation of eigenvalues given by (21) is especially simple in the case of $\varphi$ being a polynomial of $\lambda$. Precisely this particular situation was considered in all of the abovementioned references concerning Sturm-Liouville problems with spectral parameter dependent boundary conditions. For these problems the calculation of eigenvalues using our method does not present any additional difficulty compared to the parameter independent situation discussed in the preceding section.

\section{$5 \quad$ Singular problems}

As was mentioned in Remark 4, one of the solutions of the Sturm-Liouville equation can be singular and nevertheless the method presented here is still applicable. We show one such application to an interesting problem first considered in [2] and then in a number of recent publications [3, 17, 10, 11, 21, 22. We consider on the interval $(-\pi, \pi)$ the singular non-symmetric differential equation

$$
-i \varepsilon \frac{d}{d x}\left(\sin x \frac{d u}{d x}\right)-i \frac{d u}{d x}=\lambda u
$$

with $0<\varepsilon<2$ and periodic conditions at $-\pi$ and $\pi$. In spite of the fact that (22) is highly non-self-adjoint (with complex and singular coefficients) all of the eigenvalues are real [21] and the spectrum is discrete [10]. Several algorithms have appeared for approximating the eigenvalues to which we compare our results. It is known (e.g., [3]) that for each $\lambda \in \mathbb{C}$, equation (22) possesses a unique (up to scalar multiples) solution in $L^{2}(-\pi, \pi)$ which we denote by $\varphi(x, \lambda)$. This solution may be normalized by the condition $\varphi(0, \lambda)=1$. Any solution linearly independent of $\varphi$ will blow up as $x \rightarrow 0$. Also [3] $\lambda$ is an eigenvalue if and only if

$$
\varphi(-\pi, \lambda)=\varphi(\pi, \lambda)
$$

It is not difficult to rewrite equation (22) in the form (3),

$$
-i \varepsilon \frac{d}{d x}\left(\sin x\left(\tan \frac{x}{2}\right)^{1 / \varepsilon} \frac{d u}{d x}\right)=\lambda\left(\tan \frac{x}{2}\right)^{1 / \varepsilon} u \text {. }
$$


Choosing $u_{0} \equiv 1$ as a particular solution corresponding to $\lambda=0$ we immediately find that $u_{1}$ defined by (5) belongs to $L^{2}(-\pi, \pi)$, while $u_{2}$ is the singular solution blowing up as $x \rightarrow 0$. Moreover, due to the particular form of the coefficients in (23) we have from Remark 7 that

$$
\begin{aligned}
\tilde{X}^{(2 k)}(x) & =-\left(\tan \frac{x}{2}\right)^{-1 / \varepsilon} \int_{0}^{x}\left(\tan \frac{s}{2}\right)^{1 / \varepsilon} \tilde{X}^{2(k-1)}(s) d s+\int_{0}^{x} \tilde{X}^{2(k-1)}(s) d s \\
k & =1,2, \ldots
\end{aligned}
$$

Both integrals are well behaved at $x=\pi$. Moreover, the integral $\int_{0}^{x}\left(\tan \frac{s}{2}\right)^{1 / \varepsilon} d s$ can be expressed explicitly in terms of hypergeometric functions (produced for example by symbolic manipulations in Mathematica version 6). In the numerical calculation of $\widetilde{X}^{(2 k)}$ and subsequently of $u_{1}$ we may approximate the first integral above by the sum of corresponding integrals on subintervals, each one calculated as follows:

$$
\int_{x_{j-1}}^{x_{j}}\left(\tan \frac{s}{2}\right)^{1 / \varepsilon} \widetilde{X}^{2(k-1)}(s) d s \approx \tilde{X}^{2(k-1)}\left(\frac{x_{j}+x_{j-1}}{2}\right) \int_{x_{j-1}}^{x_{j}}\left(\tan \frac{s}{2}\right)^{1 / \varepsilon} d s .
$$

We show the results of application of the SPPS method in comparison with some values calculated previously. For $\varepsilon=0.5$ our values lie between those previously published. For $\varepsilon=0.1$ our results display a remarkable agreement with those of $[10$.

Eigenvalues of (22) for $\varepsilon=0.5$

\begin{tabular}{|l|c|c|c|}
\hline $\mathrm{n}$ & \multicolumn{1}{|c|}{$\lambda_{n}[22]$} & $\lambda_{n}[\underline{7}]$ & $\lambda_{n}$ SPPS \\
\hline 1 & 1.16714 & 1.167342 & 1.16723 \\
\hline 2 & 2.96821 & 2.968852 & 2.96844 \\
\hline 3 & 5.48168 & 5.483680 & 5.48268 \\
\hline 4 & 8.71272 & 8.715534 & 8.71354 \\
\hline 5 & 12.66119 & & 12.6618 \\
\hline 6 & 17.32643 & & 17.3275 \\
\hline 7 & 22.71033 & & 22.7110 \\
\hline 8 & 28.81106 & & 28.8122 \\
\hline 9 & 35.62928 & & 35.6311 \\
\hline 10 & 43.16666 & & 43.1677 \\
\hline
\end{tabular}


Eigenvalues of (22) for $\varepsilon=0.1$

\begin{tabular}{|l|c|c|c|c|}
\hline $\mathrm{n}$ & \multicolumn{1}{|c|}{$\lambda_{n}[\underline{2}]$} & $\lambda_{n}[\underline{22}]$ & $\lambda_{n}[\underline{10}]$ & $\lambda_{n}$ SPPS \\
\hline 1 & 1.0097 & 1.00940 & 1.00968 & 1.00968 \\
\hline 2 & 2.0733 & 2.07305 & 2.07334 & 2.07334 \\
\hline 3 & 3.2297 & 3.22894 & 3.22978 & 3.22978 \\
\hline 4 & 4.5012 & 4.50088 & 4.50134 & 4.50134 \\
\hline 5 & 5.8992 & 5.89968 & 5.89993 & 5.89993 \\
\hline 6 & 7.4298 & 7.43154 & 7.43194 & 7.43194 \\
\hline 7 & 9.0951 & 9.10034 & 9.10097 & 9.10097 \\
\hline 8 & 10.8945 & 10.90881 & 10.9092 & 10.9092 \\
\hline 9 & 12.8252 & 12.85742 & 12.8578 & 12.8578 \\
\hline 10 & 14.8820 & 14.94727 & 14.9478 & 14.9478 \\
\hline 15 & & & 27.5331 & 27.5331 \\
\hline 20 & & & 43.74 & 43.6923 \\
\hline
\end{tabular}

We also show the results we obtained for $\varepsilon=0.01$, for which we do not know any previous result. For this reason we include information on the accuracy of the eigenfunctions, estimated as follows. The calculated eigenfunctions $u$ were effectively substituted into the differential equation (23) by applying the corresponding integral operators which in principle should produce $\lambda u$, and the discrepancy $\delta_{1}$ at the right endpoint $\pi$ was tabulated. Then the discrepancy $\delta_{2}$ in the right boundary condition was evaluated.

Eigenvalues of (22) for $\varepsilon=0.01$

\begin{tabular}{|l|l|l|l|}
\hline $\mathrm{n}$ & $\lambda_{n}$ SPPS & \multicolumn{1}{|c|}{$\delta_{1}$} & \multicolumn{1}{c|}{$\delta_{2}$} \\
\hline 1 & 1.0001 & $1.0 \times 10^{-14}$ & $3.8 \times 10^{-16}$ \\
\hline 2 & 2.0008 & $1.5 \times 10^{-13}$ & $1.7 \times 10^{-14}$ \\
\hline 3 & 3.00269 & $3.0 \times 10^{-12}$ & $7.3 \times 10^{-14}$ \\
\hline 4 & 4.00638 & $4.9 \times 10^{-11}$ & $2.9 \times 10^{-12}$ \\
\hline 5 & 5.01243 & $6.8 \times 10^{-10}$ & $1.3 \times 10^{-11}$ \\
\hline 6 & 6.02143 & $8.8 \times 10^{-9}$ & $6.5 \times 10^{-9}$ \\
\hline 7 & 7.03393 & $3.2 \times 10^{-7}$ & $6.7 \times 10^{-8}$ \\
\hline 8 & 8.05048 & $6.2 \times 10^{-6}$ & $3.4 \times 10^{-7}$ \\
\hline 9 & 9.07162 & 0.000090 & $4.0 \times 10^{-6}$ \\
\hline 10 & 10.098 & 0.014 & 0.00027 \\
\hline 11 & 11.0223 & 10.8 & 0.0011 \\
\hline
\end{tabular}

In [11] it was proved that the set of eigenvalues of the problem tends to $\mathbb{Z}$ as $\varepsilon \rightarrow 0$. Figure 1 (where the eigenvalues have been calculated by SPPS) illustrates this assertion.

\section{Conclusions}

In the present work a new representation for solutions of the Sturm-Liouville equation is presented, which we call a spectral parameter power series (SPPS). 




Figure 1: Convergence of $\lambda_{n}$ to $n$ as $\varepsilon \rightarrow 0$.

It gives a new natural and powerful method for solving initial value, boundary value, and spectral problems. We have shown that it is applicable not only to regular problems but also to singular problems as well as to Sturm-Liouville problems with spectral parameter dependent boundary conditions. The numerical realization of the method is simple, and its ideas can be explained to undergraduate students in engineering, mathematics, and physics. One of the important advantages of the SPPS method consists in the automatic calculation of eigenfunctions together with the eigenvalues of a spectral problem. The SPPS method is competitive with the best software for numerical solution of ordinary differential equations and spectral problems currently available. The authors' goal has been to show the broad applicability and competitiveness of the method rather than to achieve spectacular numerical results; we believe that much better performance of the SPPS method can be achieved with the implementation of additional computational techniques, some of which have been mentioned in this paper.

Acknowledgements: The authors express their gratitude to CONACYT for partial support of this work as well as to Prof. Michael Levitin for attracting our attention to the problem considered in section 5 .

\section{References}

[1] Ben Amara J and Shkalikov A A 1999 A Sturm-Liouville problem with physical and spectral parameters in boundary conditions. Mathematical Notes 66, no. 2, $127-134$. 
[2] Benilov E S, O'Brien S B G and Sazonov I A 2003 A new type of instability: explosive disturbances in a liquid film inside a rotating horizontal cylinder. J. Fluid Mech. 497, 201-224.

[3] Boulton L, Levitin M and Marletta M 2008 A PT-symmetric periodic problem with boundary and interior singularities. arXiv:0801.0172 v1.

[4] Chanane B 1998 Eigenvalues of Sturm-Liouville problems using Fliess series. Applicable Analysis 69, 233-238.

[5] Chanane B 2008 Sturm-Liouville problems with parameter dependent potential and boundary conditions. J. Comput. Appl. Math. 212 , no. 2, 282-290.

[6] Child M S and Chambers A V 1988 Persistent accidental degeneracies for the Coffey-Evans potential. J. Phys. Chem 92, 3122-3124.

[7] Chugunova M and Pelinovsky D 2007 Spectrum of a non-selfadjoint operator associated with the periodic heat equation, preprint http://arxiv.org/abs/math-ph/0702100v2.

[8] Code W J and Browne P J 2005 Sturm-Liouville problems with boundary conditions depending quadratically on the eigenparameter. J. Math. Anal. Appl. 309, no. $2,729-742$.

[9] Coşkun H and Bayram N 2005 Asymptotics of eigenvalues for regular SturmLiouville problems with eigenvalue parameter in the boundary condition. J. Math. Anal. Appl. 306, no. 2, 548-566.

[10] Davies E B 2007 An indefinite convection-diffusion operator. LMS J. Comp. Math. 10, 288-306.

[11] Davies E B and Weir J 2008 Convergence of eigenvalues for a highly non-selfadjoint differential operator. arXiv:0809.0787v1.

[12] Fulton Ch T 1977 Two-point boundary value problems with eigenvalue parameter contained in the boundary conditions. Proc. Roy. Soc. Edinburgh Sect. A 77, no. 3-4, 293-308.

[13] Guillemin V and Pollack A 1974 Differential topology. Prentice-Hall, Inc., Englewood Cliffs, N.J.

[14] Kravchenko V V 2008 A representation for solutions of the Sturm-Liouville equation. Complex Variables and Elliptic Problems, 2008, v. 53, 775-789.

[15] Ledoux V 2007 Study of Special Algorithms for solving Sturm-Liouville and Schrödinger Equations, thesis Universiteit Gent

[16] Levitan B M and Sargsjan I S 1991 Sturm-Liouville and Dirac operators. Dordrecht: Kluwer Acad. Publ.

[17] Pöschel J and Trubowitz E 1987 Inverse spectral theory. Boston: Academic Press. 
[18] Paine J W, De Hoog F R and Anderssen R R 1981 Computing 26, 123-139

[19] Pryce J D 1993 Numerical solution of Sturm-Liouville problems. Clarendon Press.

[20] Walter J 1973 Regular eigenvalue problems with eigenvalue parameter in the boundary condition. Math. Z. 133, 301-312.

[21] Weir J 2008 An indefinite convection-diffusion operator with real spectrum. Applied Mathematics Letters, in press.

[22] Weir J 2008 Correspondence of the eigenvalues of a non-self-adjoint operator to those of a self-adjoint operator. arXiv:0801.4959v2.

[23] Weyl H 1910 Über gewöhnliche Differentialgleichungen mit Singularitäten und die zugehörigen Entwicklungen willkürlicher Funktionen. (German) Math. Ann. 68, no. 2, 220-269. 